\title{
miR-452-5p regulates the responsiveness of intestinal epithelial cells in inflammatory bowel disease through Mcl-1
}

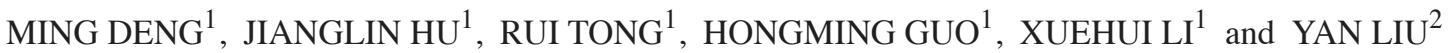 \\ ${ }^{1}$ Department of Anorectal Surgery, People's Hospital of Dongxihu District, Wuhan, Hubei 430040; \\ ${ }^{2}$ Department of Gastrointestinal Surgery, Union Hospital of Tongji Medical College, Huazhong \\ University of Science and Technology, Wuhan, Hubei 430022, P.R. China
}

Received July 8, 2020; Accepted March 17, 2021

DOI: $10.3892 / \mathrm{etm} .2021 .10245$

\begin{abstract}
Inflammatory bowel diseases (IBDs) are chronic immune disorders that occur in the intestinal tract. Previous studies have revealed that intestinal epithelial cells (IECs) play critical roles in the development of IBDs, and therapies targeting IECs hold great potential for the treatment of IBDs. However, the roles of microRNAs (miRs) in the regulation of IEC properties and whether they can be used as targets for IEC regulation and IBD treatment are largely unknown. The aim of the present study was to investigate the role of the miR-452-5p/Mcl-1 axis in the regulation of the properties of IECs during the pathology of IBD. A dextran sulfate sodium-induced mouse model of ulcerative colitis (UC) and an in vitro lipopolysaccharide-stimulated IEC-6 cell model were investigated. The results revealed that miR-452-5p expression in the IECs of the mice increased significantly upon UC induction, and the knockdown of miR-452-5p alleviated the IBD symptoms. Furthermore, the suppression of miR-452-5p downregulated the expression of the inflammatory cytokines IL-6, IL- 8 and TNF $\alpha$, and upregulated the expression of intestinal barrier-associated molecules, namely occludin, zona occludens 1 and mucin-2 in IECs in vitro and in vivo. Notably, the results indicated that miR-452-5p modulated the responses of IECs by negatively regulating the expression of Mcl-1, as the knockdown of Mcl-1 abrogated the effects of miR-452-5p suppression on IECs. The present study suggested that $\mathrm{miR}-452-5 \mathrm{p}$ regulated the responsiveness of IECs to influence the development of UC in an Mcl-1-dependent manner. These observations provide important information to improve the understanding of IBD pathogenesis and indicate
\end{abstract}

Correspondence to: Dr Yan Liu, Department of Gastrointestinal Surgery, Union Hospital of Tongji Medical College, Huazhong University of Science and Technology, 1227 Jiefang Avenue, Wuhan, Hubei 430022, P.R. China

E-mail: 1lordyliu@hotmail.com

Key words: miR-452-5p, intestinal epithelial cells, inflammatory bowel diseases, $\mathrm{Mcl}-1$ that targeting the miR-452-5p-Mcl-1 signaling axis in IECs holds potential for IBD treatment.

\section{Introduction}

Inflammatory bowel diseases (IBDs) primarily comprise Crohn's disease (CD) and ulcerative colitis (UC) (1-3). Although the pathology of IBDs has been widely investigated, the exact causes of IBDs are remain largely unknown. Currently, there are $\sim 8$ million patients with IBDs worldwide (4), and an improved understanding of such diseases should improve the efficiency of clinical therapies and benefit patients.

Intestinal epithelial cells (IECs) comprise a variety of cell types, including Paneth cells and goblet cells, and they facilitate the intestinal epithelial defense against pathogen invasion (5-8). Upon stimulation, IECs produce inflammatory cytokines, intestinal barrier-associated molecules and antimicrobial peptides to maintain intestinal homeostasis under pathophysiological conditions $(9,10)$. IEC dysfunction has been demonstrated to disrupt intestinal homeostasis and induce or aggravate the development of IBDs (9). For instance, the specific knockout of STAT3 in IECs has been shown to impair mucosal wound healing in IBD, causing mice to be highly sensitive to experimental colitis (11). Other studies have shown that the local delivery of IL-22 enhances the function of IECs in colitis and alleviates the disease, while the local delivery of IL-22-binding protein, which neutralizes IL-22 activity, significantly inhibits the restitution of IECs and limits tissue recovery following the induction of dextran sulfate sodium (DSS)-induced colitis in mice $(12,13)$. Therefore, gaining a full understanding of the regulation of IEC function is urgently necessary for the prevention and treatment of IBD.

MicroRNAs (miRNAs/miRs) are small non-coding RNA molecules that are not translated into proteins, but play critical roles in the regulation of gene expression $(14,15)$. Previous studies have revealed that miRNAs serve important roles in the development of IBD by regulating the function of cells in the intestinal tract (15). For example, in one study, miR-21 and miR-31 were shown to influence $\mathrm{T}$ cell responses in patients with IBD and animal colitis models (16). In another study, the investigators found that compared with those of healthy controls, the plasma levels of miR-199a-5p, miR-362-3p and miR-532-3p were increased in patients with $C D$ while 
those of miR-149 and miRplus-F1065 were decreased (17). Furthermore, the inhibition of certain miRNAs has been demonstrated to directly affect the induction of colitis in mice. For example, in 2,4,6-trinitrobenzenesulfonic acid (TNBS)-induced colitis in mice, anti-miR-124 treatment alleviated the disease activity index and inhibited proinflammatory cytokine expression via modulation of the aryl hydrocarbon receptor (18). In another study, the inhibition of miR-31 significantly promoted the expression of the anti-inflammatory cytokine IL-25 in the colons of mice with TNBS-induced colitis, exhibiting therapeutic effects on TNBS-induced colitis and spontaneous colitis in IL-10-deficient mice (19). However, whether miRNAs regulate the function of IECs in IBD and the underlying mechanisms remain largely unknown. Advances in this field are likely to provide new information to improve understanding of the pathology of IBD and the efficacy of IEC-based IBD therapies.

In the current study, the role of miR-452-5p in IBD was explored. It has previously been demonstrated that miR-452-5p post-transcriptionally abrogates SMAD4 expression, inhibiting the downstream gene SMAD7 (20). Also, it has been suggested that genetic variants of several SMAD family members, namely SMAD2/3/4/7, may alter the balance of differentiation between $\mathrm{T}$ helper 17 and regulatory $\mathrm{T}$ cells, resulting in the development of IBD, particularly UC (21). Additionally, the depletion of epithelial SMAD4 upregulates inflammation and promotes inflammation-associated cancer in mice with DSS-induced colitis (22). Furthermore, SMAD4 epithelial protein levels are downregulated in patients with $\mathrm{CD}$ and negatively correlated with disease activity (23). These findings suggest a key role for SMAD4 or SMAD4/SMAD7 signaling in the pathology of IBD. As miR-452-5p is associated with the upstream mechanism of SMAD4/SMAD7 signaling, we hypothesized that miR-452-5p may be involved in the progression of IBD.

In the present study, the role of the miR-452-5p/Mcl-1 axis in regulating the properties of IECs during the pathology of colitis was investigated. The findings provide new knowledge about the role of miRNAs in IBD pathogenesis and hold the potential to improve future treatments for IBDs.

\section{Materials and methods}

Mice. A total of 40 C57BL/6 male mice (6-8 weeks old; $20 \pm 2 \mathrm{~g}$ ) were purchased from Shanghai SLAC Laboratory Animal Co., Ltd. and raised at the SPF animal facility of Huazhong University of Science and Technology. The mice were housed at a constant room temperature $\left(23 \pm 2^{\circ} \mathrm{C}\right)$ and relative humidity $(50 \pm 10 \%)$ with free access to food and water in a fixed 12-h light/dark cycle. All animal experiments were performed according to the National Institutes of Health Guide for the Care and Use of Laboratory Animals (7th edition, revised 1996) and were approved by the Animal Experimentation Ethics Committee of Huazhong University of Science and Technology.

DSS-induced UC mouse model. A mouse model of UC was established using 3\% DSS (3 g/100 ml; molecular weight 36,000-50,000; MP Biomedicals, LLC) dissolved in sterile distilled water and provided ad libitum from experimental days 1 to 5 . The DSS solution was prepared fresh every 2 days to ensure its effects were maintained. The same volume of double-distilled water (DDW) was used as a control (28 mice in the UC group and 12 mice in the control group). On day 5, the mice were sacrificed by $\mathrm{CO}_{2}$ inhalation; the mice were placed in a transparent polycarbonate euthanasia chamber covered with an acrylic lid, with ports for gas inlet and outlet. The air in the chamber was replaced with $\mathrm{CO}_{2}$ at a rate of $30 \%$ chamber volume $/ \mathrm{min}$. The distal colon $(2 \mathrm{~cm})$ was removed and processed for histological examination and protein isolation.

Adenovirus (Ad) vector treatment. To explore the effects of miR-452-5p in IECs in the UC mouse model, Ad-packaged vectors (Ad-anti-miR-452-5p and Ad-Mock) were constructed using the pAdEasy/Track-CMV adenovirus vector. A scrambled sequence served as the control. Viral packaging was performed by Shanghai Kelei Biotechnology Co, Ltd. The Ad vectors (100 $\mu \mathrm{l}$ normal saline containing 5.0x10 ${ }^{8}$ active viral particles) were intracolonically administered to the mice 2 days prior to the administration of DSS. This involved administering the adenovirus intracolonically via an $8-\mathrm{cm}$ polyethylene tube through the anus under mild anesthesia (intraperitoneal injection of thiopental, $40 \mathrm{mg} / \mathrm{kg}$ ). The mice were maintained in a head-down position for $\sim 1 \mathrm{~min}$ to prevent expulsion of the solution. The mice were divided into 3 groups: Ad-Mock group (mice injected with Ad-Mock and DDW; $\mathrm{n}=12$ ), Ad-Mock+DSS group (mice injected with Ad-Mock and DSS; $n=14$ ), and Ad-anti-miR-452-5p+DSS group (mice injected with Ad-anti-miR-452-5p and DSS; $n=14$ ).

Evaluation of experimental UC. The symptoms of IBD, including loss of body weight, occult blood, and diarrhea, were recorded every day. To calculate the disease index, evaluations of body weight loss in comparison with initial body weight, stool consistency and rectal bleeding were performed. The scores were assigned as follows: For body weight loss: No weight loss, 0 ; body weight loss $1-5 \%, 1$; body weight loss 6-10\%, 2; body weight loss 11-20\%, 3; and body weight $>20 \%$, 4. For stool consistency: Well-formed pellets, 0; pasty and semiformed stools, 2; and liquid stools, 4. For rectal bleeding: No blood, 0; positive bleeding, 2; and gross bleeding, 4 . The total scores were the sum of the scores in the three categories.

Histological analysis. Hematoxylin and eosin (H\&E) staining was performed to evaluate the pathology of the mouse model of UC. In brief, colon tissues from normal mice and UC model mice were collected and fixed in PBS containing 4\% paraformaldehyde at room temperature for $48 \mathrm{~h}$. After dehydration with sequential 95 and $100 \%$ ethanol followed by xylene, the tissues were embedded in paraffin and sectioned at a thickness of $6 \mu \mathrm{m}$ with a microtome (SM2500; Leica Microsystems $\mathrm{GmbH})$. These sections were stained with hematoxylin $(0.5 \%)$ for $5 \mathrm{~min}$, followed by eosin $(0.5 \%)$ for $5 \mathrm{~min}$ at room temperature, and examined by light microscopy (Olympus Corporation).

Reverse transcription-quantitative PCR (RT-qPCR) analysis. Total RNA was extracted from the isolated IECs using TRIzol reagent (Thermo Fisher Scientific, Inc.) and reverse transcribed 
into cDNA at $55^{\circ} \mathrm{C}$ for 10 min using a PrimeScript RT-PCR kit (Takara Biotechnology Co., Ltd.). The mRNA levels were quantified by qPCR using a 7500 ABI Prism system (Applied Biosystems; Thermo Fisher Scientific, Inc.) using a SYBR-Green Master Mix kit (Thermo Fisher Scientific, Inc.). The reaction conditions were as follows: $94^{\circ} \mathrm{C}$ for $3 \mathrm{~min}$, followed by 30 cycles at $94^{\circ} \mathrm{C}$ for $45 \mathrm{sec}, 57^{\circ} \mathrm{C}$ for $45 \mathrm{sec}$ and $72^{\circ} \mathrm{C}$ for $45 \mathrm{sec}$, and final extension at $72^{\circ} \mathrm{C}$ for $10 \mathrm{~min}$. The primers used for qPCR analysis were as follows: Mouse TNF $\alpha$ forward, 5'-ACTGAACTTCGGGGTGATCG-3' and reverse, 5'-GTTTGCTACGACGTGGGCTA-3'; mouse IL-8 forward, 5'-AGAGCTTGAGTGTGACGCC-3' and reverse, 5'-CCAGGTCAGTTAGCCTTGCC-3'; mouse IL-6 forward, 5'-GTCCTTCCTACCCCAATTTCCA-3' and reverse, 5'-TAA CGCACTAGGTTTGCCGA-3'; mouse occludin (OCLN) forward, 5'-TTTCAGGTGAATGGGTCACCG-3' and reverse, 5'-GCTCCCAAGATA AGCGAACCT-3'; mouse mucin 2 (MUC-2) forward, 5'TTGTCACCTTCGATGGGCTC-3' and reverse, 5'-TCTCGTGGCGCACAATAAGT-3'; mouse zona occludens 1 (ZO-1) forward, 5'-AGAAAAAGAATGCAC AGAGTTGT-3' and reverse, 5'-GAAATCGTGCTGATG TGCCA-3'; mouse myeloid cell leukemia 1 (Mcl-1) forward, 5'-CACGTACAGGACCTAGAAGGC-3' and reverse, 5'-TAG TTTGGTGGCTGGAGCTTT-3'; mouse Bax forward, 5'-CTG CAGAGGATGATTGCTG-3' and reverse, 5'-ATCAGCAAA CATGTCAGCT-3'; mouse Bcl-2 forward, 5'-CTGAGTACC TGAACCGGCAT-3' and reverse, 5'-TTGTGGCCCAGGTAT GCAC-3'; mouse GAPDH forward, 5'-TCTTTTGCGTCG CCAGCC-3' and reverse, 5'-CCATGGGTGGAATCATAT TGGAAC-3'; mouse miR-452-5p, forward 5'-UGUUUGCAG AGGAAAC-3' and reverse, 5'-AACGCTTCACGAATTTGC GT-3'; U6 forward, 5'-AACGCTTCACGAATTTGCGT-3' and reverse, 5'-CTCGCTTCGGCAGCACA-3'. The expression of miR-452-5p was normalized to U6 and the other mRNAs were normalized to GAPDH using the $2^{-\triangle \Delta C q}$ method (24).

IEC isolation. IECs were isolated as previously described (25). Briefly, colon samples were collected, and the contents were removed by washing with PBS 3 times. After the removal of Peyer's patches and mesenteric fat, the samples were cut into $1-\mathrm{cm}$ pieces and placed into Hank's Balanced Salt Solution (Thermo Fisher Scientific, Inc.) supplemented with $1 \mathrm{mM}$ dithiothreitol, 0.5 mM EDTA and 10\% FBS (Gibco; Thermo Fisher Scientific, Inc.), and shaken at $37^{\circ} \mathrm{C}$ for $30 \mathrm{~min}$. The cell suspension was collected, filtered through a $40-\mu \mathrm{m}$ strainer to remove debris, centrifuged at $4^{\circ} \mathrm{C}$ for $5 \mathrm{~min}$ at $700 \mathrm{x} \mathrm{g}$, and then resuspended in $25 \%$ Percoll. The cell suspension was gently added to the top of $40 \%$ Percoll in a $15-\mathrm{ml}$ tube. The tubes were centrifuged at $400 \mathrm{x} \mathrm{g}$ at room temperature for $20 \mathrm{~min}$, and IECs were collected from the interphase for analysis by RT-qPCR and western blotting.

Cell treatment and transfection. IEC- 6 cells (BeNa Culture Collection) were cultured in McCoy's 5A medium (Sigma-Aldrich; Merck KGaA) supplemented with $10 \%$ FBS (Thermo Fisher Scientific, Inc.), $100 \mathrm{U} / \mathrm{ml}$ penicillin G potassium and $100 \mu \mathrm{g} / \mathrm{ml}$ streptomycin at $37^{\circ} \mathrm{C}$ in a humidified atmosphere with $5 \% \mathrm{CO}_{2}$. LPS $(1 \mu \mathrm{g} / \mathrm{ml}$; Sigma-Aldrich; Merck KGaA) was used to stimulate IEC-6 cells to induce inflammation for $4 \mathrm{~h}$ at $37^{\circ} \mathrm{C}$. Silencing of Mcl-1 was performed by cloning short hairpin RNA (shRNA) oligonucleotides targeting Mcl-1 into the pCMV vector (Shanghai GenePharma Co., Ltd.), and its scrambled negative control (sh-NC) was also purchased from Shanghai GenePharma Co., Ltd. Mock refers to untreated cells. The miR-452-5p mimics (miR-452-5p; 5'-UGU UUGCAGAGGAAACUGAGAC-3') and non-targeting control mimics (NC mimics; 5'-UUUGUACUACACAAAAGUACU G-3'), miR-452-5p inhibitor (anti-miR-452-5p; 5'-GUCUCA GUUUCCUCUGCAAACA-3') and its non-targeting control (anti-miR-NC; 5'-CAGUACUUUUGUGUAGUACAAA-3') were also purchased from Shanghai GenePharma Co., Ltd. IEC- 6 cells were seeded into 24 -well plates at a density of $2.0 \times 10^{4}$ cells/well, following which $50 \mathrm{nM}$ synthetic oligonucleotides or $2 \mu \mathrm{g}$ vectors were transfected into the cells using Lipofectamine ${ }^{\circledR} 2000$ (Thermo Fisher Scientific, Inc.) for $48 \mathrm{~h}$ at $37^{\circ} \mathrm{C}$ according to the manufacturer's instructions. Cells were collected after $48 \mathrm{~h}$ for further experiments.

Western blotting. Colon tissues and in vitro cultured cells were collected, and the samples were lysed with RIPA lysis buffer (Beyotime Institute of Biotechnology). containing protease inhibitors at a mass/volume ratio of $100 \mathrm{mg} / \mathrm{ml}$. Protein was then extracted and protein concentration was detected using a BCA kit (Beyotime Institute of Biotechnology). At least $10 \mu \mathrm{g}$ protein extracts were used for $10 \%$ SDS-PAGE. After running the gels, the protein samples were transferred onto nitrocellulose membranes, followed by blocking with $5 \%$ skimmed milk at $4^{\circ} \mathrm{C}$ overnight. The membranes were sequentially incubated with the following primary antibodies: Mcl-1 (1:500; cat. no. ab32087; Abcam), TNFa (1:1,000; cat. no. ab183218; Abcam), IL-8 (1:1,000; cat. no. AMM02601G; Santa Cruz Biotechnology, Inc.), IL-6 (1:1,000; cat. no. ab229381; Abcam), OCLN (1:1,000; cat. no. ab216327; Abcam), OCLN (1:1,000; cat. no. ab221547; Abcam), MUC-2 (1:1,000; cat. no. ab272692; Abcam) and $\beta$-actin (1:2,000; cat. no. AMM04710G; Santa Cruz Biotechnology, Inc.) overnight at $4^{\circ} \mathrm{C}$, followed by incubation with HRP-conjugated goat anti-rabbit IgG H\&L antibodies (1:2,000; cat. no. ab6721; Abcam) for $1 \mathrm{~h}$ at room temperature. GAPDH served as an internal control. After washing with TBS with $0.1 \%$ Tween 20 three times, the immunoreactive bands were detected using enhanced chemiluminescence (Pierce; Thermo Fisher Scientific, Inc.) and analyzed using ImageJ v1.8.0 software (National Institutes of Health).

Flow cytometry. After transfection for $48 \mathrm{~h}$, an Annexin V-fluorescein isothiocyanate (FITC)/propidium iodide (PI) Apoptosis Detection kit (BD Biosciences) was used to analyze the apoptosis of the IEC- 6 cells. The cells were collected with trypsin, washed with PBS, and resuspended in $500 \mu 1$ HEPES buffer solution (Sigma-Aldrich; Merck KGaA). The cells were then incubated with $5 \mu \mathrm{l}$ Annexin V-FITC and $5 \mu 1 \mathrm{PI}$ at room temperature for $15 \mathrm{~min}$ in the dark. Finally, a FACSVerse ${ }^{\mathrm{TM}}$ flow cytometer with FACSCanto II FACP Array ${ }^{\mathrm{TM}}$ software (v.3.0; both BD Biosciences) was used to analyze apoptosis.

Luciferase reporter assay. The binding sites for miR-452-5p and Mcl-1 were predicted using the starBase 3.0 website (http://starbase.sysu.edu.cn/). The luciferase reporter assay was performed as previously described (26). The 3'-untranslated version (3'UTR) of Mcl-1 containing the predicted 
wild-type (Wt) miR-452-5p-binding sequence and a mutant (Mut) version of this sequence were amplified by Shanghai GenePharma Co., Ltd. and inserted into the luciferase reporter gene of the pmirGLO vector (Promega Corporation), which produced the reporter plasmids Mcl-1-Wt and Mcl-1-Mut, respectively. IEC-6 cells were co-transfected with miR-452-5p mimics, anti-miR-452-5p or Mock (NC mimics) and the Mcl-1-Wt/Mut luciferase constructs (100 ng) and the Renilla luciferase control plasmid pRL-SV40 plasmid (2 ng) using Lipofectamine 2000. pRL-SV40 was used to standardize the transfection efficiency and exclude experimental errors caused by differences in transfection efficiencies. The ratio of the firefly and Renilla reniformis luciferase activities was used as an indicator of the luciferase activity. Luciferase activity in the IECs was analyzed using a Dual-Luciferase ${ }^{\circledR}$ reporter assay system (Promega Corporation) after $24 \mathrm{~h}$ of incubation.

Statistical analysis. All experiments were performed at least three times. Data are presented as the mean \pm SEM. Differences between two groups were evaluated by two-tailed Student's t-test. Statistical significance among three or more groups was assessed by one-way ANOVA followed by post hoc Dunnett's test (for comparisons with one control) or Tukey's test (for comparisons among various groups). Statistical significance for the disease activity index was analyzed using the Mann-Whitney test. The correlation between miR-452-5p and Mcl-1 expression was analyzed using Spearman's correlation test. $\mathrm{P}<0.05$ was considered to indicate a statistically significant difference.

\section{Results}

miR-452-5p regulates the functions of IECs during the development of UC. The expression of miR-452-5p was assessed in IECs isolated from healthy mice and mice with DSS-induced UC. H\&E staining was also conducted. The results indicated that there was severe mucosal injury in DSS-induced mice, characterized by epithelial cell disruption, massive bowel edema and distorted architecture of crypts (Fig. 1A). Based on the RT-qPCR results, it was observed that compared with healthy colon-derived IECs, the IECs in DSS-induced colitis tissue expressed significantly higher levels of miR-452-5p (Fig. 1B). This indicates that miR-452-5p may act as a regulator of IBD development and exert its effects by regulating the function of IECs. To confirm this hypothesis, mice were treated with Ad-Mock or Ad-anti-miR-452-5p. After confirming the downregulation of miR-452-5p in the IECs of mice to which Ad-anti-miR-452-5p was administered (Fig. 2A), the susceptibility of the mice to DSS-induced UC was assessed. It was found that anti-miR-452-5p exhibited beneficial effects on UC model mice, as revealed by a decreased disease activity index (Fig. 2B) and attenuated loss of colon length (Fig. 2C). Notably, the inhibition of miR-452-5p in IECs significantly restrained the expression of the inflammatory cytokines TNF- $\alpha$, IL- 6 and IL- 8 , and maintained normal expression levels of the integrity-associated molecules OCLN, ZO-1 and MUC-2 in the IECs of the mice exposed to DSS (Fig. 2D and E). Collectively, these results indicate that miR-452-5p participates in the development of IBD by regulating the function of IECs.
miR-452-5p negatively regulates the expression of Mcl-1 in IECs. The starBase analysis revealed that numerous mRNAs, including Mcl-2, contain putative binding sites for miR-452-5p. Based on the potential role of Mcl-1 in IBD (27), the relationship between Mcl-1 and miR-452-5p in colitis was explored. The results revealed that the expression of Mcl-1 was significantly downregulated in the IECs of mice with DSS-induced colitis compared with those from the healthy controls (Fig. 3A-C). Spearman's correlation analysis (Fig. 3D) revealed that miR-452-5p and Mcl-1 were negatively correlated, suggesting that miR-452-5p negatively regulates the expression of Mcl-1 in IECs. Consistent with this, anti-miR-452-5p significantly promoted the expression of Mcl-1 in the IECs of the Ad-miR-452-5p group compared with the Ad-Mock group (Fig. 3E and F).

To further confirm the effects of miR-452-5p on Mcl-1 expression in IECs, miR-452-5p mimics and anti-miR-452-5p were transfected into IEC- 6 cells. The transfection efficiencies of anti-miR-452-5p and miR-452-5p mimics in these IECs were tested using RT-qPCR. The results demonstrated that the expression of miR-452-5p was decreased by anti-miR-452-5p and elevated by miR-452-5p mimics (Fig. S1A). Further analysis revealed that miR-452-5p mimics significantly inhibited the expression of Mcl-1 in the IEC-6 cell line, while the knockdown of miR-452-5p had the opposite effect (Fig. 4A and B). Furthermore, a potential binding site of miR-452-5p and the 3'UTR of Mcl-1 was predicted using the starBase database. To determine whether miR-452-5p directly regulates $\mathrm{Mcl}-1$, luciferase reporter assays were performed in cells containing the full-length 3'UTR of Wt Mcl-1. The miR-452-5p mimic reduced the activity of the Mcl-1-Wt luciferase reporter whereas anti-miR-452-5p increased luciferase reporter activity. Furthermore, mutagenesis of the miR-452-5p binding site in the Mcl-1 3'UTR abolished these effects (Fig. 4C and D). These results demonstrate that miR-452-5p directly targets Mcl-1.

miR-452-5p/Mcl-1 axis influences the responsiveness of IECs upon activation. The roles of the miR-452-5p/Mcl-1 axis in the regulation of IEC function under activated conditions were further analyzed. Consistent with the in vivo data, the RT-qPCR results showed that increased levels of miR-452-5p were associated with decreased levels of Mcl-1 upon LPS stimulation (Fig. 5A). Further experiments were conducted by transfecting the IECs with sh-Mcl-1, the transfection efficiency of which was tested by RT-qPCR. The results indicated that Mcl-1 expression was successfully decreased by sh-Mcl-1 (Fig. S1B). As shown in Fig. 5B-H, treatment with LPS significantly promoted the levels of TNF $\alpha$, IL- 6 and IL-8, inhibited the levels of OCLN, ZO-1 and MUC-2, and increased apoptosis compared with those in the control group. The inhibition of miR-452-5p significantly inhibited the expression of TNF $\alpha$, IL- 6 and IL-8 induced by LPS activation, while the knockdown of Mcl-1 abrogated the effects of miR-452-5p inhibition on these inflammatory cytokines in IECs (Fig. 5B and D). Furthermore, the inhibition of miR-452-5p reversed the downregulation of OCLN, ZO-1 and MUC-2 induced by LPS stimulation, and Mcl-1 knockdown abolished these effects (Fig. 5C and E). As Mcl-1 also has effects on apoptosis, whether the miR-452-5p/Mcl-1 axis modulates IEC apoptosis was examined. The results 
A

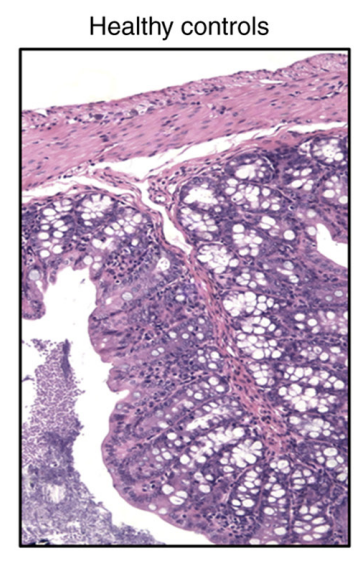

Ulcerative colitis

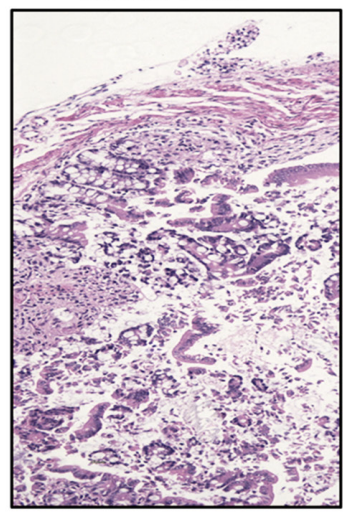

B

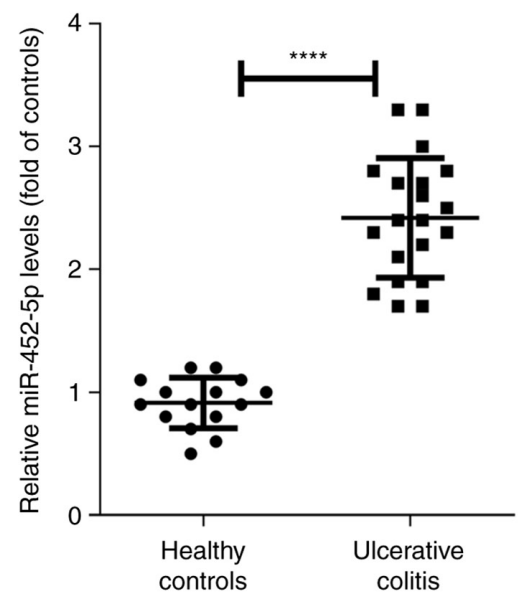

Figure 1. Expression of miR-452-5p in IECs increases during inflammatory bowel disease. (A) Histological analysis of colon tissue from healthy mice and mice with ulcerative colitis by hematoxylin and eosin staining. (B) Expression of miR-452-5p in IECs isolated from the mice. ${ }^{* * * *} \mathrm{P}<0.0001$. miR, microRNA; IECs, intestinal epithelial cells.

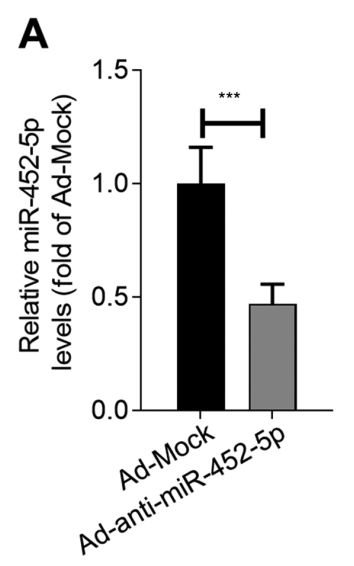

D

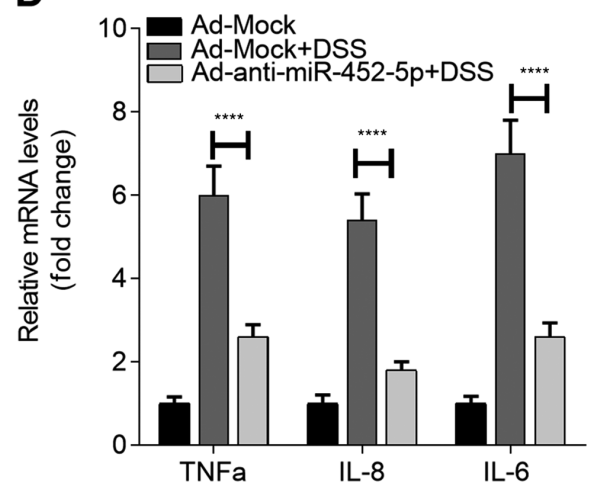

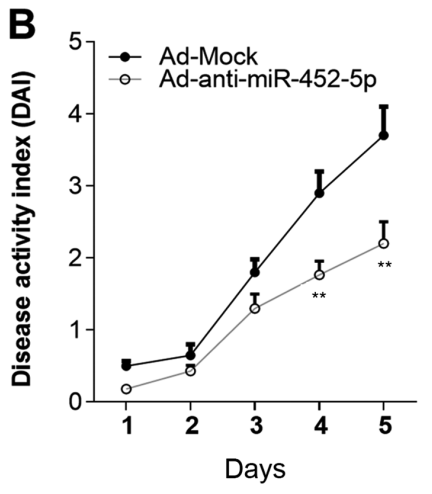

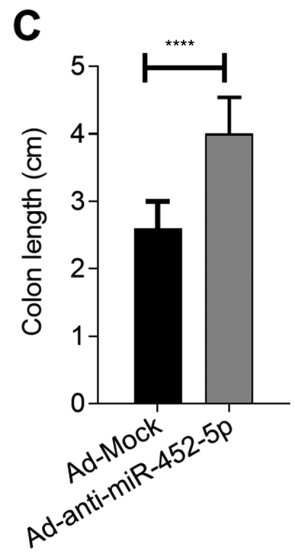

E

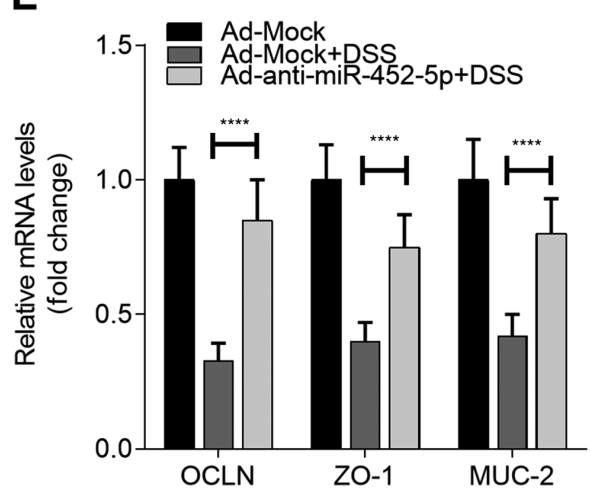

Figure 2. Inhibition of miR-452-5p alleviates the symptoms of IBD. (A) The efficiency of miR-452-5p knockdown in the IECs of mice. (B) Effect of miR-452-5p knockdown on the development of IBD. (C) Effect of miR-452-5p knockdown on colon shortening during IBD. Effects of miR-452-5p knockdown on the RNA expression of (D) inflammatory cytokines and (E) integrity-associated molecules in IECs during IBD. ${ }^{* *} \mathrm{P}<0.01,{ }^{* * *} \mathrm{P}<0.001$ and ${ }^{* * * * *} \mathrm{P}<0.0001$ vs. Ad-Mock or as indicated. miR, microRNA; IBD, inflammatory bowel disease; IECs, intestinal epithelial cells; OCLN, occludin; ZO-1, zona occludens 1; MUC-2, mucin-2; Ad, adenovirus; DDW, double-distilled water; DSS, dextran sulfate sodium.

showed that miR-452-5p knockdown inhibited IEC apoptosis and Mcl-1 knockdown attenuated this effect, indicating that miR-452-5p promotes the apoptosis of IECs by inhibiting Mcl-1 expression (Fig. 5F and G). These data indicate that miR-452-5p regulates the responsiveness of IECs to LPS activation in an Mcl-1-dependent manner.
Collectively, these data demonstrate that miR-452-5p negatively regulates the expression of Mcl-1 in IECs, exacerbating the progression of colitis. This information will help with understanding the pathology of IBD and may facilitate improvements in the efficacy of clinical strategies. 
A
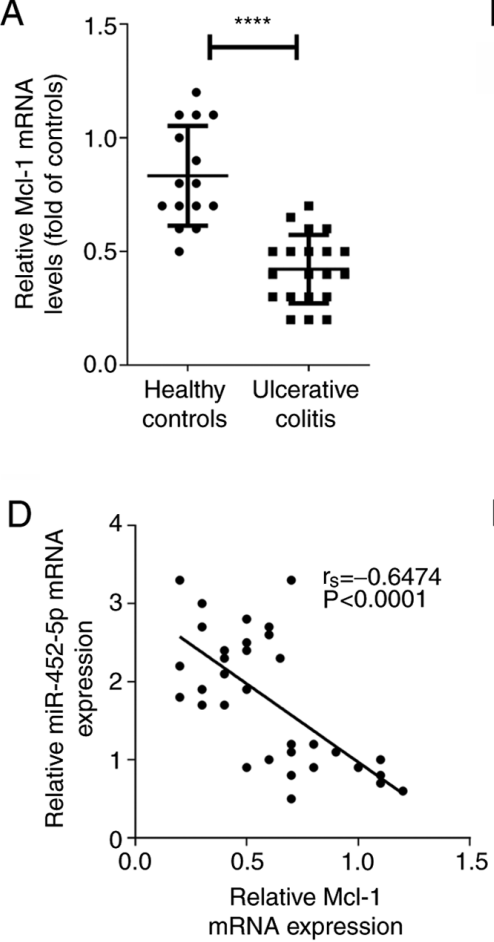

B

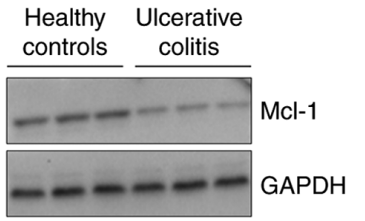

$\mathrm{E}$

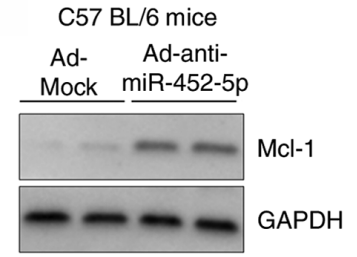

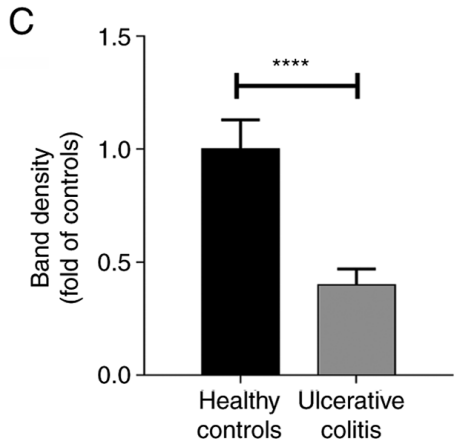

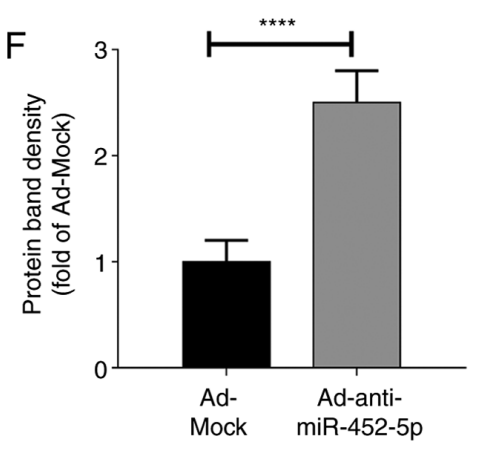

Figure 3. miR-452-5p expression is negatively correlated with Mcl-1 expression in IECs. (A) mRNA expression of Mcl-1 in IECs isolated from healthy mice and mice with ulcerative colitis. Protein levels of Mcl-1 in IECs derived from the mice. (B) Representative blots and (C) quantified results are presented. (D) Correlation between miR-452-5p and Mcl-1 in the IECs of the mice. Effects of miR-452-5p inhibition on the (E) protein and (F) mRNA expression of Mcl-1. ${ }^{* * * * *} \mathrm{P}<0.0001$. miR, microRNA; Mcl-1, myeloid cell leukemia 1; IECs, intestinal epithelial cells; Ad, adenovirus.
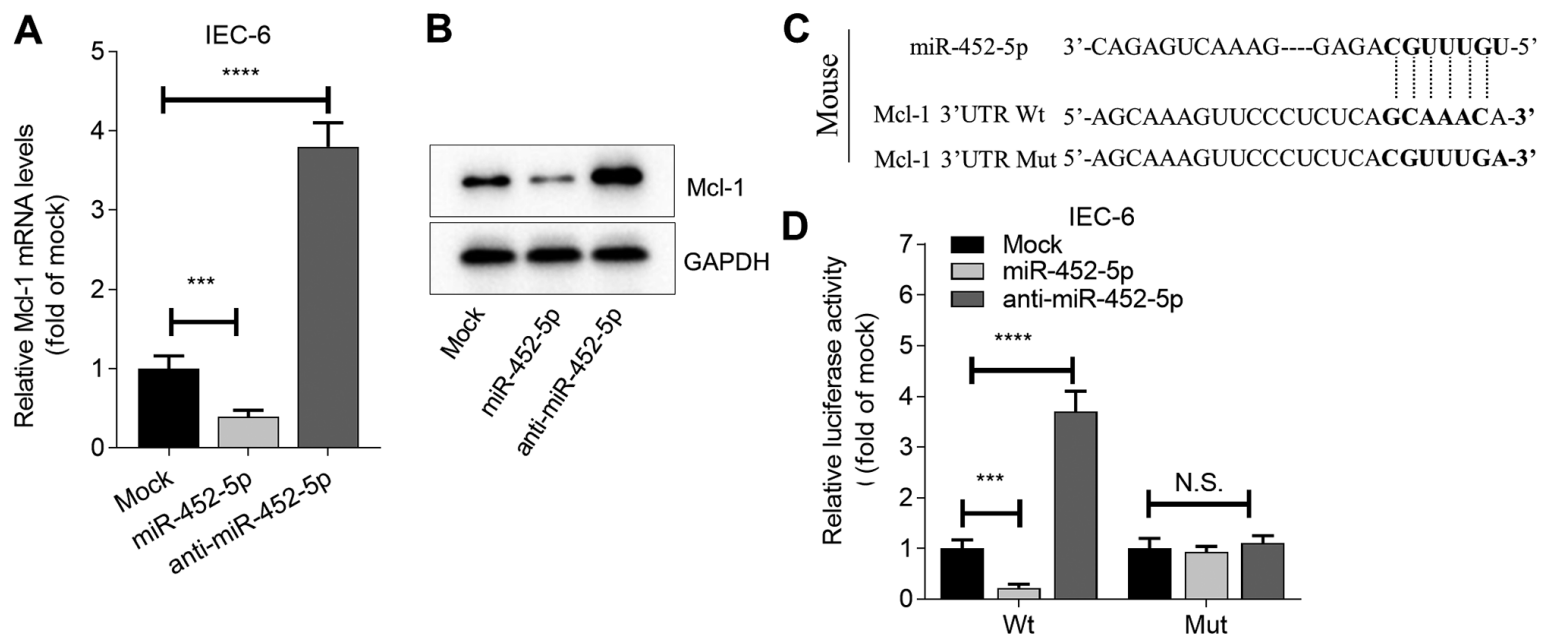

Figure 4. miR-452-5p directly regulates the expression of Mcl-1 in IECs. Transfection with miR-452-5p mimic or anti-miR-452-5p regulates the (A) mRNA levels and (B) protein levels of Mcl-1 in IEC-6 cells. (C) Predicted binding sites between miR-452-5p and Mcl-1. (D) Analysis of the binding of miR-452-5p with the Mcl-1 mRNA 3'UTR using luciferase reporter assays. ${ }^{* * *} \mathrm{P}<0.001$ and ${ }^{* * * * *} \mathrm{P}<0.0001$. miR, microRNA; Mcl-1, myeloid cell leukemia 1; IECs, intestinal epithelial cells; 3'UTR, 3'-untranslated region; Wt, wild-type; Mut, mutant; N.S., not significant.

\section{Discussion}

IBDs are chronic inflammatory disorders that affect intestinal tissues, and fully understanding IBD pathology is critical for the development of efficient strategies to combat these conditions (1). Previous studies have demonstrated that IECs serve critical roles in the pathogenesis of IBDs $(28,29)$. In the present study, it was demonstrated that upon the induction of UC in mice using DSS, IECs expressed increased levels of inflammatory cytokines, namely TNF $\alpha$, IL- 8 and IL-6, and reduced levels of intestinal integrity-associated molecules, namely OCLN, ZO-1 and MUC-2. These observations indicate that the function of the IEC barrier is largely impaired during IBD progression, and these dysfunctional IECs also participate in the initiation and maintenance of chronic inflammation. Thus, developing therapies to target IECs holds great potential for the treatment of IBD. 
A

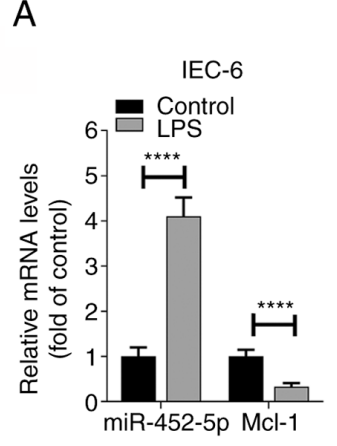

B

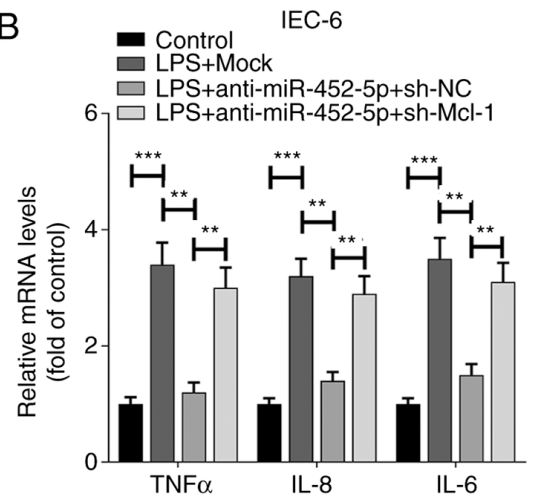

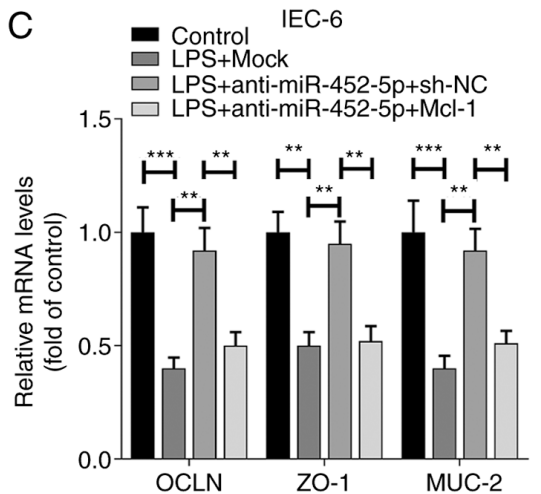

D

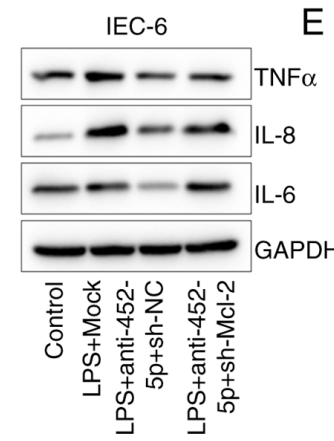

G
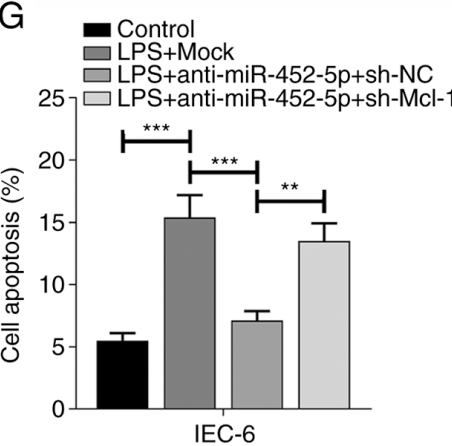

E IEC-6

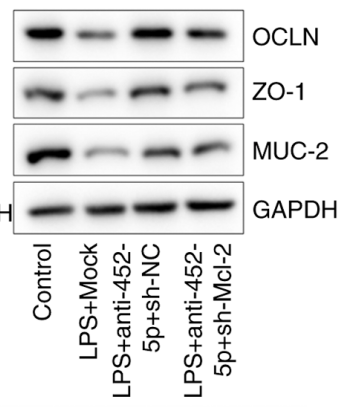

$\mathrm{H}$

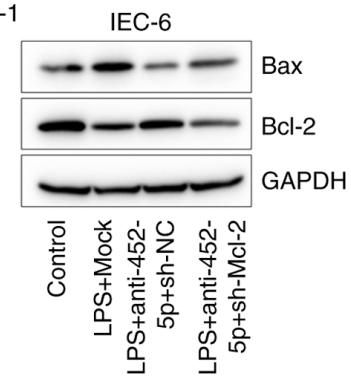

F

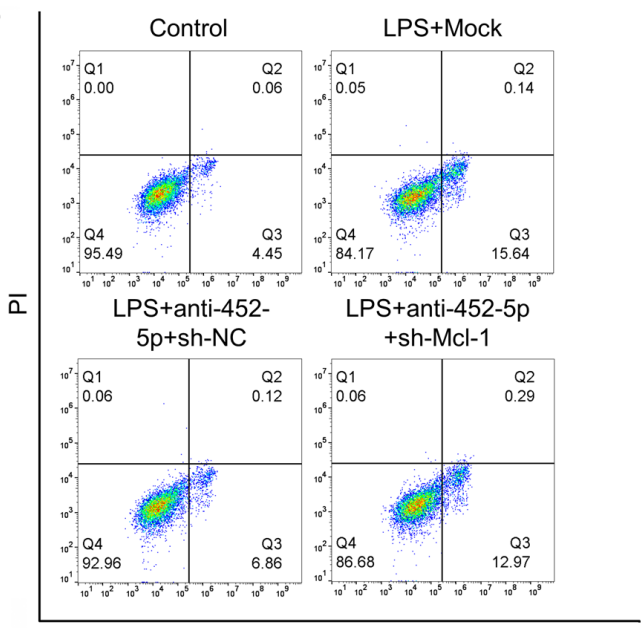

Annexin V-FITC

Figure 5. miR-452-5p/Mcl-1 signaling axis regulates the responsiveness of IECs to LPS stimulation. (A) mRNA expression of miR-452-5p and Mcl-1 in IEC-6 cells with or without LPS stimulation. (B-E) Effects of the miR-452-5p-Mcl-1 axis on the mRNA expression of (B) inflammatory cytokines and (C) integrity-associated molecules and the protein expression of (D) inflammatory cytokines and (E) integrity-associated molecules in IEC-6 cells upon LPS activation. Effects of the miR-452-5p-Mcl-1 axis on apoptosis in IEC-6 cells in response to LPS activation; (F) representative plots and (G) quantified data are presented. (H) Effects of the miR-452-5p-Mcl-1 axis on the expression of Bax and Bcl-2 in IEC-6 cells in response to LPS activation. ${ }^{* * *} \mathrm{P}<0.01 ;{ }^{* * * *} \mathrm{P}<0.001 ;{ }^{* * * * *} \mathrm{P}<0.0001$. miR, microRNA; Mcl-1, myeloid cell leukemia 1; IECs, intestinal epithelial cells; LPS, lipopolysaccharide; OCLN, occludin; ZO-1, zona occludens 1; MUC-2, mucin-2; sh, short hairpin; NC, negative control.

In the present study, RT-qPCR analysis revealed that the expression of miR-452-5p was upregulated in the mouse model of UC and in LPS-treated IECs. The data also demonstrated that miR-452-5p is an important regulator of the expression of inflammatory cytokines and intestinal integrity-associated molecules. Furthermore, the knockdown of miR-452-5p significantly alleviated the symptoms of IBD in the mouse model. These results indicate that miR-452-5p plays a key role in the pathogenesis of IBD. Thus, further investigations to elucidate the function of other miRNAs in IECs or other types of intestinal cells are likely to provide more potential therapeutic targets for IBD treatment.

Mcl-1 is a member of the Bcl-2 family and is associated with apoptosis (30). A previous study indicated that the intestinal pathology associated with IEC-specific Mcl-1 deficiency exhibited hallmark features of IBD, including barrier dysfunction, chronic inflammation, increased IEC apoptosis, hyperproliferation and impaired IEC differentiation, demonstrating the crucial role of Mcl-1 in the maintenance of intestinal homeostasis (27). Mcl-1 has also been reported to be downregulated in tissue samples from patients with fibrotic CD (31), suggesting a potential role of Mcl-1 in IBD. Additionally, Mcl-1 has been shown to be involved in the regulation of the LPS-induced inflammatory response. For example, in one study, Mcl-1 overexpression alleviated LPS-induced IL-1 $\beta$, IL-6, IL-8, and TNF $\alpha$ expression in ATDC5 murine chondrogenic cells (32). In another study, Mcl-1 knockdown promoted LPS-induced apoptosis and the release of inflammatory cytokines in C28/I2 
chondrocytes (33). In the present study, bioinformatics analysis predicted that Mcl-1 contains a binding site for miR-452-5p, and a luciferase reporter assay confirmed this binding capacity. Furthermore, the overexpression of miR-452-5p inhibited the expression of Mcl-1 in IECs, and the knockdown of Mcl-1 abrogated the effects of anti-miR-452-5p on the expression of inflammatory cytokines and integrity-associated molecules. Although the present study confirmed that miR-452-5p regulates the expression of $\mathrm{Mcl}-1$, further investigation into whether other molecules and signaling pathways are regulated by miR-452-5p and participate in the miR-452-5p-mediated regulation of IECs is merited.

By comparing the levels of IEC-expressed miR-452-5p between normal mice and mice with experimental UC, the present study indicated that miR-452-5p may represent a promising target for IBD treatment. The knockdown of miR-452-5p in the IECs of the mice demonstrated that miR-452-5p inhibition significantly relieved the symptoms of IBD. Furthermore, the data suggested that miR-452-5p promoted inflammation and impaired intestinal integrity by negatively regulating the expression of Mcl-1 in IECs, as the knockdown of Mcl-1 abrogated the effects of miR-452-5p knockdown in IECs in vitro. Overall, the present study demonstrated that miR-452-5p regulates the responsiveness of IECs in IBD by inhibiting Mcl-1 expression. These findings provide new information on the pathogenesis of IBD and may be of benefit to future clinical treatments.

\section{Acknowledgements}

Not applicable.

\section{Funding}

No funding was received.

\section{Availability of data and materials}

The datasets used and/or analyzed during the current study are available from the corresponding author on reasonable request.

\section{Authors' contributions}

MD performed the experiments and analyzed the data. JH, RT, HG and XL helped to perform the experiments and analyzed the data. YL designed the study, wrote the manuscript and provided material support. MD and YL confirmed the authenticity of all the raw data. All the authors read and approved the final manuscript.

\section{Ethics approval and consent to participate}

The study was approved by the Animal Experimentation Ethics Committee of Huazhong University of Science and Technology.

\section{Patient consent for publication}

Not applicable.

\section{Competing interests}

The authors declare that they have no competing interests.

\section{References}

1. Uhlig $\mathrm{HH}$ and Powrie F: Translating immunology into therapeutic concepts for inflammatory bowel disease. Annu Rev Immunol 36: 755-781, 2018.

2. Ungaro R, Mehandru S, Allen PB, Peyrin-Biroulet L and Colombel JF: Ulcerative colitis. Lancet 389: 1756-1770, 2017.

3. Torres J, Mehandru S, Colombel JF and Peyrin-Biroulet L: Crohn's disease. Lancet 389: 1741-1755, 2017.

4. GBD 2017 Inflammatory Bowel Disease Collaborators: The global, regional, and national burden of inflammatory bowel disease in 195 countries and territories, 1990-2017: A systematic analysis for the Global Burden of Disease Study 2017. Lancet Gastroenterol Hepatol 5: 17-30, 2020.

5. Hegyi P, Maleth J, Walters JR, Hofmann AF and Keely SJ: Guts and gall: Bile acids in regulation of intestinal epithelial function in health and disease. Physiol Rev 98: 1983-2023, 2018.

6. Saavedra PHV, Huang L, Ghazavi F, Kourula S, Vanden Berghe T, Takahashi N, Vandenabeele P and Lamkanfi M: Apoptosis of intestinal epithelial cells restricts Clostridium difficile infection in a model of pseudomembranous colitis. Nat Commun 9: 4846, 2018.

7. VanDussen KL, Stojmirovic A, Li K, Liu TC, Kimes PK, Muegge BD, Simpson KF, Ciorba MA, Perrigoue JG, Friedman JR, et al: Abnormal small intestinal epithelial microvilli in patients with Crohn's disease. Gastroenterology 155: 815-828, 2018

8. Geng H, Bu HF, Liu F, Wu L, Pfeifer K, Chou PM, Wang X, Sun J, Lu L, Pandey A, et al: In inflamed intestinal tissues and epithelial cells, interleukin 22 signaling increases expression of H19 long noncoding RNA, which promotes mucosal regeneration. Gastroenterology 155: 144-155, 2018.

9. Peterson LW and Artis D: Intestinal epithelial cells: Regulators of barrier function and immune homeostasis. Nat Rev Immunol 14: 141-153, 2014.

10. Turner JR: Intestinal mucosal barrier function in health and disease. Nat Rev Immunol 9: 799-809, 2009.

11. Pickert G, Neufert C, Leppkes M, Zheng Y, Wittkopf N, Warntjen M, Lehr HA, Hirth S, Weigmann B, Wirtz S, et al: STAT3 links IL-22 signaling in intestinal epithelial cells to mucosal wound healing. J Exp Med 206: 1465-1472, 2009.

12. Sugimoto K, Ogawa A, Mizoguchi E, Shimomura Y, Andoh A, Bhan AK, Blumberg RS, Xavier RJ and Mizoguchi A: IL-22 ameliorates intestinal inflammation in a mouse model of ulcerative colitis. J Clin Invest 118: 534-544, 2008.

13. Wang Y, Mumm JB, Herbst R, Kolbeck R and Wang Y: IL-22 increases permeability of intestinal epithelial tight junctions by enhancing claudin-2 expression. J Immunol 199: 3316-3325, 2017.

14. Garofalo M and Croce CM: microRNAs: Master regulators as potential therapeutics in cancer. Annu Rev Pharmacol Toxicol 51: 25-43, 2011.

15. Visone R, Petrocca F and Croce CM: Micro-RNAs in gastrointestinal and liver disease. Gastroenterology 135: 1866-1869, 2008.

16. Mohammadnia-Afrouzi M, Hosseini AZ, Khalili A, Abediankenari S, Amari A, Aghili B and Nataj HH: Altered microRNA expression and immunosuppressive cytokine production by regulatory $\mathrm{T}$ cells of ulcerative colitis patients. Immunol Invest 45: 63-74, 2016.

17. Wu F, Guo NJ, Tian H, Marohn M, Gearhart S, Bayless TM, Brant SR and Kwon JH: Peripheral blood microRNAs distinguish active ulcerative colitis and Crohn's disease. Inflamm Bowel Dis 17: 241-250, 2011.

18. Zhao Y, Ma T, Chen W, Chen Y, Li M, Ren L, Chen J, Cao R, Feng Y, Zhang H and Shi R: MicroRNA-124 promotes intestinal inflammation by targeting aryl hydrocarbon receptor in Crohn's disease. J Crohns Colitis 10: 703-712, 2016.

19. Shi T, Xie Y, Fu Y, Zhou Q, Ma Z, Ma J, Huang Z, Zhang J and Chen J: The signaling axis of microRNA-31/interleukin-25 regulates Th1/Th17-mediated inflammation response in colitis. Mucosal Immunol 10: 983-995, 2017. 
20. Zhai W, Li S, Zhang J, Chen Y, Ma J, Kong W, Gong D, Zheng J, Xue W and Xu Y: Sunitinib-suppressed miR-452-5p facilitates renal cancer cell invasion and metastasis through modulating SMAD4/SMAD7 signals. Mol Cancer 17: 157, 2018.

21. Yamashita A, Inamine $T$, Suzuki S, Fukuda S, Unoike M, Kawafuchi Y, Machida H, Isomoto H, Nakao K and Tsukamoto K: Genetic variants of SMAD2/3/4/7 are associated with susceptibility to ulcerative colitis in a Japanese genetic background. Immunol Lett 207: 64-72, 2019.

22. Means AL, Freeman TJ,Zhu J, Woodbury LG, Marincola-Smith $P$ Wu C, Meyer AR, Weaver CJ, Padmanabhan C, An H, et al: Epithelial Smad4 deletion up-regulates inflammation and promotes inflammation-associated cancer. Cell Mol Gastroenterol Hepatol 6: 257-276, 2018.

23. Klausen P, Karstensen JG, Coskun M, Săftoiu A, Vilmann P Cowland JB and Riis LB: SMAD4 protein expression is downregulated in ileal epithelial cells from patients with Crohn's disease with significant inverse correlation to disease activity. Gastroenterol Res Pract 2018: 9307848, 2018.

24. Livak KJ and Schmittgen TD: Analysis of relative gene expression data using real-time quantitative PCR and the 2(-Delta Delta C(T)) method. Methods 25: 402-408, 2001.

25. Roulis M, Armaka M, Manoloukos M, Apostolaki M and Kollias G: Intestinal epithelial cells as producers but not targets of chronic TNF suffice to cause murine Crohn-like pathology. Proc Natl Acad Sci USA 108: 5396-5401, 2011.

26. Ito $\mathrm{Y}$, Inoue $\mathrm{A}$, Seers $\mathrm{T}$, Hato $\mathrm{Y}$, Igarashi $\mathrm{A}$, Toyama $\mathrm{T}$, Taganov KD, Boldin MP and Asahara H: Identification of targets of tumor suppressor microRNA-34a using a reporter library system. Proc Natl Acad Sci USA 114: 3927-3932, 2017.
27. Healy ME, Boege Y, Hodder MC, Böhm F, Malehmir M, Scherr AL, Jetzer J, Chan LK, Parrotta R, Jacobs K, et al: MCL1 is required for maintenance of intestinal homeostasis and prevention of carcinogenesis in mice. Gastroenterology 159: 183-199, 2020.

28. Ramanan $D$ and Cadwell K: Intrinsic defense mechanisms of the intestinal epithelium. Cell Host Microbe 19: 434-441, 2016

29. Odenwald MA and Turner JR: The intestinal epithelial barrier: A therapeutic target? Nat Rev Gastroenterol Hepatol 14: 9-21, 2017.

30. Kotschy A, Szlavik Z, Murray J, Davidson J, Maragno AL, Le Toumelin-Braizat G, Chanrion M, Kelly GL, Gong JN, Moujalled DM, et al: The MCL1 inhibitor S63845 is tolerable and effective in diverse cancer models. Nature 538: 477-482, 2016.

31. Nijhuis A, Curciarello R, Mehta S, Feakins R, Bishop CL, Lindsay JO and Silver A: MCL-1 is modulated in Crohn's disease fibrosis by miR-29b via IL-6 and IL-8. Cell Tissue Res 368: 325-335, 2017.

32. Wang Y and Kong D: MicroRNA-136 promotes lipopolysaccharide-induced ATDC5 cell injury and inflammatory cytokine expression by targeting myeloid cell leukemia 1. J Cell Biochem 119: 9316-9326, 2018.

33. Zhao C, Wang Y, Jin H and Yu T: Knockdown of microRNA-203 alleviates LPS-induced injury by targeting MCL-1 in C28/I2 chondrocytes. Exp Cell Res 359: 171-178, 2017.

This work is licensed under a Creative Commons Attribution-NonCommercial-NoDerivatives 4.0 International (CC BY-NC-ND 4.0) License. 